\title{
El análisis de visibilidad en la evaluación de impacto ambiental de nuevas construcciones
}

\author{
Analysis of visibility in the assessment of the \\ environmental impact of new constructions
}

$\underline{\text { I. Otero }}^{(*)}$, E. Varela(*), S. Mancebo(*), A. Ezquerra(*)

RESUMEN

Hoy en día es necesario reconciliar el desarrollo urbano con el equilibrio ecológico y adoptar un urbanismo con base territorial amplia, para controlar la localización y regulación de los usos y actividades. Dentro de este contexto, el trabajo que se expone en el presente artículo, se planteó como objetivo último la localización de nuevas áreas urbanas de baja densidad en el entorno del Corredor del Henares de Guadalajara (España). Los modelos de capacidad e impacto diseñados al efecto consideran como uno de sus componentes más importantes la calidad visual del paisaje, que se basa, a su vez, en un estudio exhaustivo de la visibilidad del territorio. El objeto de los análisis de visibilidad es determinar las áreas visibles desde cada punto o conjunto de puntos, bien simultáneamente o en secuencia, con vistas a la posterior evaluación de la medida en que cada área contribuye a la percepción del paisaje y a la obtención de ciertos parámetros globales que permitan caracterizar un territorio en términos visuales. Siguiendo la línea enunciada, el trabajo trata de realizar una aportación en el campo de los estudios de visibilidad, mediante el diseño de un modelo de visibilidad, posteriormente programado e implementado en SIG (Sistemas de Información Geográfica); se describe el proceso seguido para realizar esta tarea, centrándose en las dificultadas que se presentaron y su resolución. El análisis de visibilidad desarrollado constituyó un punto importante tanto en el modelo de capacidad de acogida de la actividad como en el modelo del impacto que ésta puede producir en el medio. La construcción de un modelo de visibilidad permite valorar de forma objetiva la visibilidad del territorio desde todos los puntos de interés.

113-84

Palabras clave: visibilidad, planificación, SIG, capacidad, impacto.
Informes de la Construcción Vol. 61, 515, 67-75, julio-septiembre 2009 ISSN: 0020-0883 eISSN: 1988-3234 doi: 10.3989/ic.09.014

\section{SUMMARY}

Nowadays there is a need to take account of the ecological balance in urban development, and to adopt a type of urban planning which is spread over a broad territorial base in order to control the localisation and regulation of uses and activities. In view of this requirement, the objective of the work described in the present article is to propose locations for nine low-density urban areas in the surroundings of the Corredor del Henares in Guadalajara (Spain). One of the most significant components of the capacity and impact models designed for this purpose is the visual quality of the landscape, which is based on an exhaustive study of the visibility of the territory. The object of analysing the visibility is to determine which areas can be seen from each point or set of points, either simultaneously or in sequence, in order to assess the degree to which each area contributes to the perception of the landscape, and to obtain certain global parameters which allow a territory to be characterised in visual terms. By extension of this objective, the aim of the work is to contribute to the field of visibility studies by designing a visibility model which is subsequently programmed and implemented in GIS (Geographical Information System). We describe the process used to perform this task, with particular emphasis on the difficulties which arose and how they were resolved. The visibility analysis we developed was an important element both in the model for the capacity of acceptance of the activity, and in the model for assessing the possible impact of the activity on the environment. The construction of a visibility model allows an objective assessment of the visibility of the territory from all the points of interest.

Keywords: visibility, planning, GIS, Capacity, Impact. 


\section{INTRODUCCIÓN}

Como resultado de los cambios en los valores ambientales, la valoración social de las actividades constructivas también ha evolucionado; así las obras públicas y los nuevos núcleos urbanizados han pasado a entenderse como elementos de deterioro del paisaje (1); el creciente interés por la ecología y el medio ambiente se extiende también al paisaje.

Actualmente las áreas urbanas han experimentado un importante crecimiento; aunque los patrones de crecimiento no hayan sido consistentes a lo largo del tiempo y en las distintas áreas geográficas, hoy día es necesario realizar un seguimiento de este desarrollo urbano con el objetivo de soslayar los posibles problemas ambientales derivados del desarrollo urbanístico y del incremento del tráfico que a él se asocia (2). Es necesario, en fin, reconciliar el desarrollo urbano con el equilibrio ecológico y adoptar un urbanismo con base territorial amplia, para controlar la localización y regulación de los usos y actividades.

Dentro de este contexto, el trabajo que se expone en el presente artículo, se planteó como objetivo último la localización de nuevas áreas urbanas de baja densidad en el entorno del Corredor del Henares de Guadalajara (España). Los modelos de capacidad e impacto diseñados al efecto consideran como uno de sus componentes más importantes la calidad visual del paisaje, que se basa, a su vez, en un estudio exhaustivo de la visibilidad del territorio (2); y éste es precisamente el aspecto en el cual se centra el artículo. El paisaje es un factor ambiental constituyente del inventario ambiental en las evaluaciones de impacto ambiental de nuevas obras (infraestructuras, urbanizaciones...), incluyendo diversos aspectos como las condiciones de visibilidad (3).

La mayoría de los enfoques aplicados al análisis visual del paisaje conceden gran importancia a la determinación de las áreas de visibilidad desde los distintos puntos de vista (4); algunos autores señalan la necesidad de su establecimiento previo para determinar después las características de estas zonas o áreas vistas (5).

La conveniencia de precisar las limitaciones físicas del territorio respecto a la percepción visual ha sido puesta de manifiesto principalmente por los arquitectos paisajistas (6) y ya desde 1931 existen métodos manuales para producir mapas de visibilidad por medio de esquemas de campo (7), aunque este tipo de análisis sólo pudo enfocarse de forma sistemática a partir de los últimos años de la década de los sesenta con la utilización masiva del ordenador en los estudios de planificación.

El objeto del análisis visual del paisaje es determinar las áreas visibles desde cada punto o conjunto de puntos, bien simultáneamente o en secuencia, con vistas a la posterior evaluación de la medida en que cada área contribuye a la percepción del paisaje y a la obtención de ciertos parámetros globales que permitan caracterizar un territorio en términos visuales (8). Sus aplicaciones son múltiples: van desde la planificación ambiental, a la selección de la ubicación óptima de los puntos de vista o de las luces de referencia en la costa, a analizar el impacto de las construcciones humanas en el paisaje. Otra aplicación importante es la planificación experimental para las campañas de observación del comportamiento de animales salvajes en condiciones de campo (9). Por ello, la mayoría de los métodos de evaluación del impacto visual han estado relacionados con el examen del tamaño de la reducción del impacto de un desarrollo por el terreno en el que esté situado (10).

\subsection{Determinación de la cuenca visual}

Dada una superficie del terreno y un punto de vista, el problema clásico de la visibilidad es detectar la porción de terreno visible desde dicho punto (11), esto es, la cuenca visual.

El método clásico para obtener la cuenca visual de un punto consiste en trazar una dirección visual sobre un mapa, de la cual se obtendrá el perfil transversal calculando las intersecciones con las curvas de nivel. Posteriormente se trazan rayos visuales hacia el perfil de modo que al alejarse del punto de observación se vayan obteniendo pendientes que han de ser cada vez mayores para que se mantenga la visibilidad. Se dibujan en el mapa base los puntos de comienzo y fin de las zonas de sombra (áreas no visibles desde un punto). Finalmente, se gira la dirección visual hasta barrer la zona que interese. La mayor limitación estriba en la dificultad de incorporar restricciones de visibilidad debidas a formaciones vegetales o construcciones humanas. Es por ello deseable, tanto en este método como en todos los realizados en gabinete, completar y perfeccionar la determinación con un repaso in situ, corrigiendo posibles desviaciones (8).

Así, otra metodología es la observación directa in situ, desarrollada por Litton (12) en 1973 en la que el observador se traslada al punto cuya cuenca visual se quiere determinar con un mapa de la zona al que se irán transfiriendo los límites visuales de su observación. Esta técnica es rápida y permite una mayor compenetración del observador con el 
terreno. Como inconveniente cabe señalar la muy variable fiabilidad de la determinación en función de las características personales del operador y de la zona.

Los primeros métodos analíticos desarrollados para medir la visibilidad de sitios individuales deben atribuirse a Tandy (15), Hebblethwaite (13) y Weddle (14) al principio de la década de los 70. El método de Tandy (15) es el más subjetivo ya que precisa visitar la zona de estudio para encontrar puntos focales de observación desde los que mirar al sitio. Van Der Ham y Iding (16) desarrollaron ideas similares durante la misma época en Holanda. Esta aproximación fue llevada un paso más allá por Weddle y Pickard (6 y 14), que trazaron secciones topográficas y líneas de vista a intervalos de $10^{\circ}$ alrededor de los sitios propuestos para el desarrollo de una central nuclear en el estuario del Dee, Chesshire. El problema de este método es que sólo se dibujaron unos pocos perfiles topográficos, y su construcción puede ser muy laboriosa. Sin embargo, permite identificar zonas sombra y ofrece algunas indicaciones de la zona de influencia visual.

Hebblethwaite (13) desarrolló una técnica más rápida usando un mapa de contorno y dos plantillas de plástico, una de ellas, con una línea recta horizontal que servirá para marcar la dirección visual y, la otra, con líneas horizontales paralelas equidistantes que representarán las distintas alturas con una equidistancia igual a la del mapa. Este método es descrito por completo por Clark et al. del Department of the Environment en 1976(17-19).

Aunque todos estos métodos fueron propuestos en su origen para la evaluación de sÓlo un sitio, claramente el método de Hebblethwaite (13) en particular podía usarse para comparar el tamaño de las zonas de influencia de múltiples sitios. Así podrían compararse las propuestas de desarrollos a gran escala en varias localizaciones.

\subsection{Métodos informatizados}

Existen dos procedimientos automáticos básicos de uso habitual para hallar la cuenca visual de un punto:

1. Cuenca visual por rayos.

2. Cuenca visual por cuadrículas.

En el primer procedimiento el proceso de búsqueda se organiza por medio de rayos, que recorren desde el origen o punto de observación y barren el área de estudio. En cada rayo se marcan los puntos visibles y no visibles comparando la pendiente de la recta que une cada punto en cuestión con el punto de observación, con las calculadas para puntos anteriores.

En el segundo método, para cada cuadrícula del territorio que se halle dentro del círculo cuyo radio es el alcance, se realiza el test de visibilidad de forma similar al caso anterior. Se une el punto de observación con el centro de la cuadrícula en cuestión y se va recorriendo esa recta comparando las alturas de la propia recta con las altitudes del terreno. El dibujo de la cuenca visual puede efectuarse en este caso por medio de una impresora, aunque más que un dibujo suele ser sólo un esquema (8).

Los primeros desarrollos en análisis de visibilidad fueron simplemente la informatización directa de las técnicas de Weddle y Hebblethwaite(6) (13), incluyendo el dibujo de secciones topográficas y líneas de vista. Se han realizado muchos programas para hacer esto, uno relativamente simple está disponible en MacDougall (20).

Pero en esta línea el desarrollo más importante lo constituyó el programa VIEWIT diseñado en 1975 por Travis et al. (21), que fue desarrollado anteriormente por Elsner (44). El programa "traza" una dirección visual, obtiene el perfil y determina las células visibles y las invisibles. Cuando se han barrido todos los orígenes requeridos, la memoria reservada a cada célula contiene el número de veces que cada célula es visible desde el conjunto de puntos de observación. El resultado se cartografía automáticamente imprimiendo en la posición de cada célula ese número de veces $\mathrm{o}$, transformando los números en escalas de distinta intensidad de impresión. Posteriormente Anderson et al. (22) produjeron un programa de soporte para mejorar la entrada de datos y para extender su uso.

Paralelamente el programa desarrollado por la School of Design de la Universidad de Harvard (23) para el modelo de calidad visual opera de forma ligeramente distinta. Para conseguir un algoritmo de búsqueda más eficiente separa el área que rodea la célula de origen en ocho octantes que son barridos por una serie de rayos. El usuario puede seleccionar los octantes en los que quiere realizar la búsqueda, así como el número de rayos. El programa permite también cartografiar el uso del suelo de las células que se ven y organizar la búsqueda en función de los usos, bien de la célula origen, bien de las células buscadas.

Más adelante se desarrollaron un conjunto de programas similares al VIEWITs por parte de Aylward y Turnbull (24); se realizaron para definir el tamaño de la zona de influencia visual usando un método de cálculo por 
rayo e interpolación descrito en el artículo de 1977.

La segunda línea de avance consistió en tomar el modelo básico de terreno y mejorar los programas de modo que se pueda ver la superficie del terreno desde cualquier punto por encima, $\mathrm{o}$, incluso, en la superficie del modelo (10). Este trabajo fue, una vez más, completado por el United States Department of Forestry (21); Tlusty (25) y también por Aylward y Turnbull (24). En 1984, McAulay (26) produjo un algoritmo mucho más refinado. Felleman (27) en su programa PREVIEW y Aylward y Turnbul (24), incluyen la superposición de figuras del paisaje en la superficie del terreno. Algunos aspectos más avanzados de estos gráficos de ordenador están descritos por Marshall et al. (28).

Las soluciones al cálculo de la cuenca visual dependen de la naturaleza del mapa digital disponible (9). Para representaciones del terreno como una superficie poliédrica se han propuesto varias soluciones (29).

En el caso de mapas de elevación del terreno raster MDT (Modelo Digital del Terreno) se han desarrollado numerosas soluciones (11) pero no tienen en cuenta los aspectos estocásticos.

En 1996 Sansoni (30) desarrolló una nueva técnica para determinar la visibilidad del paisaje, de forma probabilística; este método no usa un MDT construido sobre una malla regular como otros modelos previos. Por el contrario, el uso de líneas de contorno se considera como una lista de vértices, caminos y elevación relativa.

Como alternativa Anile et al. en 2003 (9), basándose en un nuevo concepto de visibilidad definido en un contexto "fuzzy", han elaborado un conjunto de programas y funciones organizadas en dos aplicaciones con una interfaz gráfica de usuario consistente y un completo soporte para el intercambio con bases de datos externas y Sistemas de Información Geográfica (SIG).

\subsection{Intervisibilidad}

El concepto de intervisibilidad y el índice de intervisibilidad se introdujeron en 1971 en el Coventry-Solihull-Warwickshire Sub-Regional Study (31). El método original manual se basaba en una malla de puntos con datos de altura, cuya finalidad era calcular un valor numérico para cada cuadrado de la malla, y el número de cuadrados que se podían ver desde cada uno de ellos (aquí está la idea de intervisibilidad).

La medida de la visibilidad resulta muy útil a la hora de establecer zonas de impac- tos visuales máximos y mínimos y en los problemas concretos de localización de actividades. En este último caso funciona reversiblemente, posibilitando la localización de las actividades que se desean más visibles y recíprocamente ocultando en las zonas menos visibles aquellas actividades necesarias para el territorio pero no deseadas visualmente (32).

Kent, en 1979, informatizó este proceso (33). Trabajando sobre cuadrados de la red de puntos de altura, el programa calcula primero un índice de visibilidad para cada cuadrado. Luego, se calcula el grado de exposición visual de cada cuadrado, y la combinación de los dos índices da un índice general de intervisibilidad para cada cuadrado. El programa ha seguido refinándose y mejorándose.

La mayoría de los análisis descritos anteriormente sólo son útiles para un único sitio o para la comparación de cierto número de sitios. Estos análisis han sido clasificados de acercamiento de "resolución de problema" (14). Sin embargo, existe la necesidad de analizar la visibilidad a la escala más general de región o planificación de estructuras, a las que Weddle se refiere como "acercamiento territorial". Los primeros métodos aplicables a escala regional se describen en Felleman $(1979,1982)(27,34)$ y Ramos et al. (1976) (35). Posteriormente, tanto Kent en 1979 (33) como Blanco et al. en 1982 (36) usaron métodos de análisis multivariable que pueden usarse en el proceso de planificación regional.

En 1978 De Veer y Burrough (37) y Burrough y De Veer en 1984 (38) describen un método informatizado basado en la elaboración de una gran base de datos en la que la información de visibilidad es sólo una parte. El análisis de visibilidad se contrasta con otros y el producto final es un mapa base en el que se superponen dos círculos concéntricos a las dos distancias críticas de percepción de Van der Ham y Iding (16). Los nuevos elementos del paisaje también pueden modelizarse de este modo, y se pueden comparar visibilidades entre diferentes lugares (10).

Posteriormente se desarrollaron los primeros programas de ese tipo para microordenadores, por ejemplo, el programa de perspectiva de Nickerson para el ordenador personal Hewlett-Packard (39). Las rutinas de BASIC para calcular diagramas de perspectiva se presentaron en 1983 en MacDougall (20).

Sin embargo, el análisis espacial de la estructura y patrones de la visibilidad del paisaje está peor estudiada que la intervisibilidad entre estructuras. 
Más recientemente, en 2000, Miller (40) ha elaborado un modelo para calcular el área desde la que los diferentes tipos de cobertura son visibles a lo largo de amplias áreas geográficas. Este método, usa una red regular para ofrecer un medio para comparar la visibilidad entre los sitios, que extiende los trabajos previos para incluir la evaluación de la visibilidad de los elementos de la cobertura del terreno, y sus cambios en el tiempo.

Analizando los métodos enunciados, se deduce que la definición de intervisibilidad es muy variable (41), no por la dificultad del concepto, sino por los diferentes enfoques que se han usado para mitigar la laboriosidad de su cálculo y que pueden resumirse como sigue:

- Investigar sólo una parte de las unidades que podrían ser visibles, como el modelo antes mencionado (23).

- Reducir la distancia máxima de visibilidad. Es el método más empleado y se suele concretar, cuando se emplean mallas, en la investigación de las células adyacentes (35). - Realizar la búsqueda sólo para unos cuantos puntos de observación. La selección de estos puntos se puede hacer por muestreo aleatorio de acuerdo con la propia naturaleza del terreno (12); o con arreglo a criterios de accesibilidad y posible número de personas en el punto de observación (42).

Por último comentar que en todos los análisis de visibilidad las unidades próximas a los límites del estudio presentan el problema de su interacción visual con áreas próximas no pertenecientes a la zona estudiada. La forma más elegante de resolver estos problemas es ampliar el área de estudio, en una zona de profundidad igual a la distancia máxima de visibilidad a lo largo de todo su perímetro.

Otra posible solución es la ampliación del inventario a las zonas colindantes con el área de estudio, pero con mucho menor detalle: utilizando, por ejemplo, unidades o mallas más amplias, restringiendo el número de parámetros inventariados para cada unidad o combinando ambas formas (8). Actualmente, es necesaria la programación de modelos de visibilidad dentro de los Sistemas de Información Geográfica. El presente artículo expone un método desarrollado en esta línea.

\section{MATERIAL Y MÉTODOS}

El modelo de visibilidad que aquí se resume se desarrolló, como ya se ha comentado, para incluirlo en el proceso de delimitación de áreas potencialmente urbanizables y propuesta de integración de criterios ambienta- les en el desarrollo urbanístico del Corredor del Henares de Guadalajara (2).

\subsection{Zona de estudio}

Guadalajara es la provincia más al norte de la Comunidad Autónoma de Castilla-La Mancha. El Corredor del Henares es una unidad territorial de unos $50 \mathrm{~km}$ de longitud por unos $25 \mathrm{~km}$ de ancho entre Madrid y Guadalajara, que recibe su nombre del río Henares. Se trata de una zona que no presenta obstáculos naturales a la implantación de vías de comunicación y núcleos urbanos; esto, unido a su estratégica situación como eje económico y de las comunicaciones, en la región central peninsular, motiva que haya sido la receptora de núcleos históricos importantes y que constituya hoy el área de expansión natural de los centros urbanos allí asentados.

El área incluida en este estudio abarca tanto lo que en líneas anteriores se ha denominado Corredor del Henares como algunos terrenos adyacentes que se encuentran bajo su influencia. La zona de estudio comprende un total de catorce municipios entre los que se encuentra la capital de provincia. Además de las poblaciones situadas estrictamente en el Corredor del Henares, que son las de mayor tamaño, se han incluido otros municipios colindantes, de bajas densidades de población, pero que están potencialmente sujetas a un fuerte desarrollo por su proximidad a este corredor.

\subsection{Métodos}

El análisis de visibilidad, es la base para la determinación de la calidad y fragilidad visual del paisaje, que constituye un punto importante tanto en el modelo de capacidad de acogida de la actividad como en el modelo del impacto que ésta puede producir en el medio, como se muestra en el esquema 1 (pág. 50), ilustrado además por el mapa de valores finales de calidad visual del paisaje (Figura 1, pág. siguiente).

La construcción de un modelo de visibilidad permite valorar de forma objetiva la visibilidad del territorio desde todos los puntos que puedan interesar.

El sistema de información geográfica Arc-GIS 9.2, es una herramienta útil para este fin, ya que permite realizar análisis de visibilidad a través de la extensión Spatial Analyst y la orden Viewshed. Para ello, se necesita el modelo digital del terreno (MDT) y los puntos del territorio para los que se quiere calcular la cuenca visual. El estudio de visibilidad es un análisis en formato ráster; la información del 
Esquema 1

Análisis de visibilidad
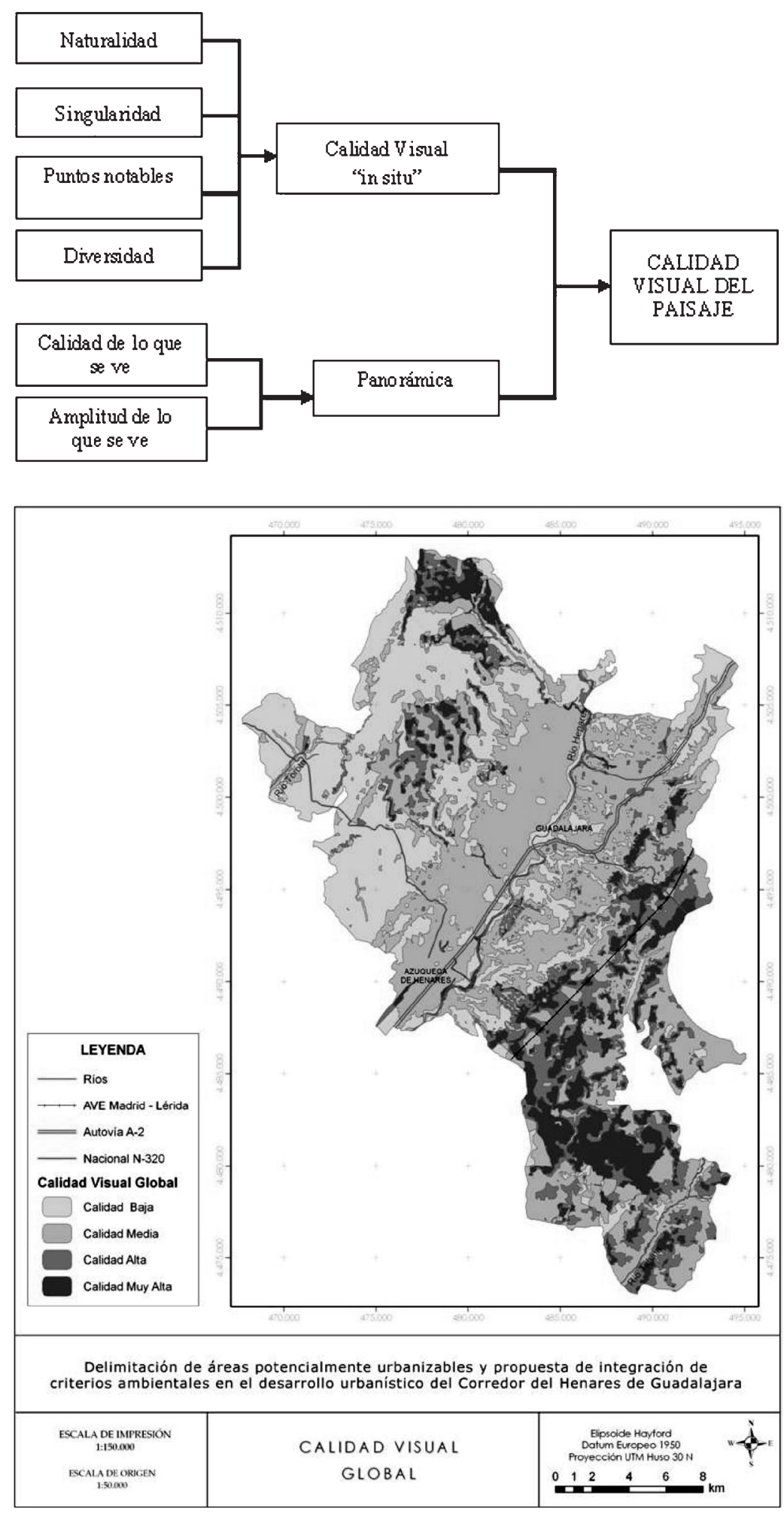

1. Calidad visual global del paisaje. Las zonas de tonalidad más oscura son las que presentan calidad más alta territorio está compartimentada en celdillas o píxeles. Los puntos desde los que se va a realizar el análisis visual han de ser elegidos cuidadosamente. Se opta por crear una malla sistemática de puntos que cubre todo el territorio. Cada punto se sitúa en el centro de una celdilla de 250 X 250 m. Este tamaño de celda permite una buena cobertura del territorio, ya que se obtienen 8.520 puntos de análisis, sin que el tiempo requerido por el programa para realizar este análisis sea excesivo.

El MDT, que es el otro elemento necesario para realizar este análisis, y que ya se había elaborado previamente, tiene un tamaño de píxel de $10 \times 10 \mathrm{~m}^{2}$. Se aumenta el tamaño de celdilla a $50 \times 50 \mathrm{~m}^{2}$ para que los cálculos sean más rápidos y sea más coherente con el área representada por cada punto. Esta transformación se hace desde Arc-Info, dentro de la extensión Grid, con la orden Resample. Esta orden realiza una interpolación para adaptar la información al nuevo tamaño de celdilla. La interpolación es de tipo cúbico, pues es la que mejor resulta, según se ha comprobado en experiencias anteriores, para este tipo de análisis.

La siguiente dificultad que se plantea es el propio análisis de visibilidad, pues la orden Viewshed de Spatial Analyst sólo puede ejecutarse para 16 puntos de forma simultánea. Como con la malla sistemática se crea un total de 8.520 puntos, sería necesario repetir la orden un número muy elevado de veces.

Por ello se opta por realizar estos análisis de visibilidad a través de Arc-Info programando en lenguaje AML (que es reconocido por ArcInfo) para que se ejecute automáticamente la visibilidad de todos los puntos (la programación realizada se puede encontrar en la web topografía.montes.upm.es).

El análisis de visibilidad se plantea inicialmente para ser empleado en el desarrollo del modelo de capacidad ya expuesto; en éste interesa conocer a qué unidad ambiental, de las definidas para dicho modelo, pertenece cada punto. Ésta es la razón por la que el programa diseñado actúa como se explica a continuación:

En primer lugar, selecciona todos los puntos pertenecientes a la unidad ambiental 1, que, de aquí en adelante, se denominarán $\mathrm{p}_{1}$. Posteriormente, calcula la visibilidad de los puntos seleccionados. Este cálculo lo realiza analizando cuántos de los puntos que constituyen la unidad $\mathrm{p}_{1}$ pueden ser vistos desde cada punto del resto del territorio. El resultado es un mapa en formato ráster donde cada celdilla tiene un valor numérico. El valor 0 corresponde a las celdillas que no ven ningún punto de $p_{1}$; el valor 1 corresponde a las celdillas que ven un punto de $\mathrm{p}_{1 .} \mathrm{Y}$ así sucesivamente.

Todo este proceso se repite para las 30 clases de puntos, que corresponden a las 30 unidades ambientales. 


\section{RESULTADOS}

En la Figura 2 se pueden ver los resultados para los puntos de la unidad ambiental 14. Las tonalidades son más oscuras en las zonas en que se divisan mayor número de puntos $\mathrm{p}_{14}$.

En el plano lejano, el campo visual aumenta, por lo que el número de píxeles que se perciben, en principio, es mayor. Sin embargo, la nitidez de lo percibido en ese plano es menor que la de lo percibido en el plano medio.

Para compensar este hecho, los resultados de visibilidad obtenidos para el plano lejano se multiplican por un factor cuyo numerador es el área del plano medio y cuyo denominador es el área del plano lejano. Los números son los siguientes:

* Área total del plano lejano $=\Pi \times\left(10^{2}-3^{2}\right)$ $=91 \Pi \mathrm{km}^{2}$.

* Área total del plano medio $=\Pi \times\left(3^{2}-1^{2}\right)$ $=8 \Pi \mathrm{km}^{2}$.

* Factor por el que se multiplica el resultado de visibilidad del plano lejano = 8/91.

Los resultados obtenidos para el plano medio y para el plano lejano compensado se suman, teniendo así un resultado global de visibilidad.

\section{CONCLUSIONES}

El programa permite fijar la altura del observador y del objeto observado así como un intervalo de distancia para el que se calcula la visibilidad.

Para la altura de observador se ha escogido la que el programa toma por defecto, es decir, 1 metro. La altura del objeto observado se ha fijado en 2 metros. La razón para esto es que el análisis que nos interesa es el inverso al que hace el programa; esto es, cuánto se ve desde cada unidad ambiental. Y por eso, lo que para el programa es el observador para nosotros será el objeto observado y viceversa. Como nuestro observador está situado en una zona urbanizada y, por tanto, ha tenido una explanación y cimentación previa, el terreno habrá sufrido una pequeña elevación que, unida a la altura propia del observador, se ha cifrado en dos metros. Los valores de visibilidad van cambiando de forma suave para cada zona del territorio. Sólo hay una pequeña excepción a esto en

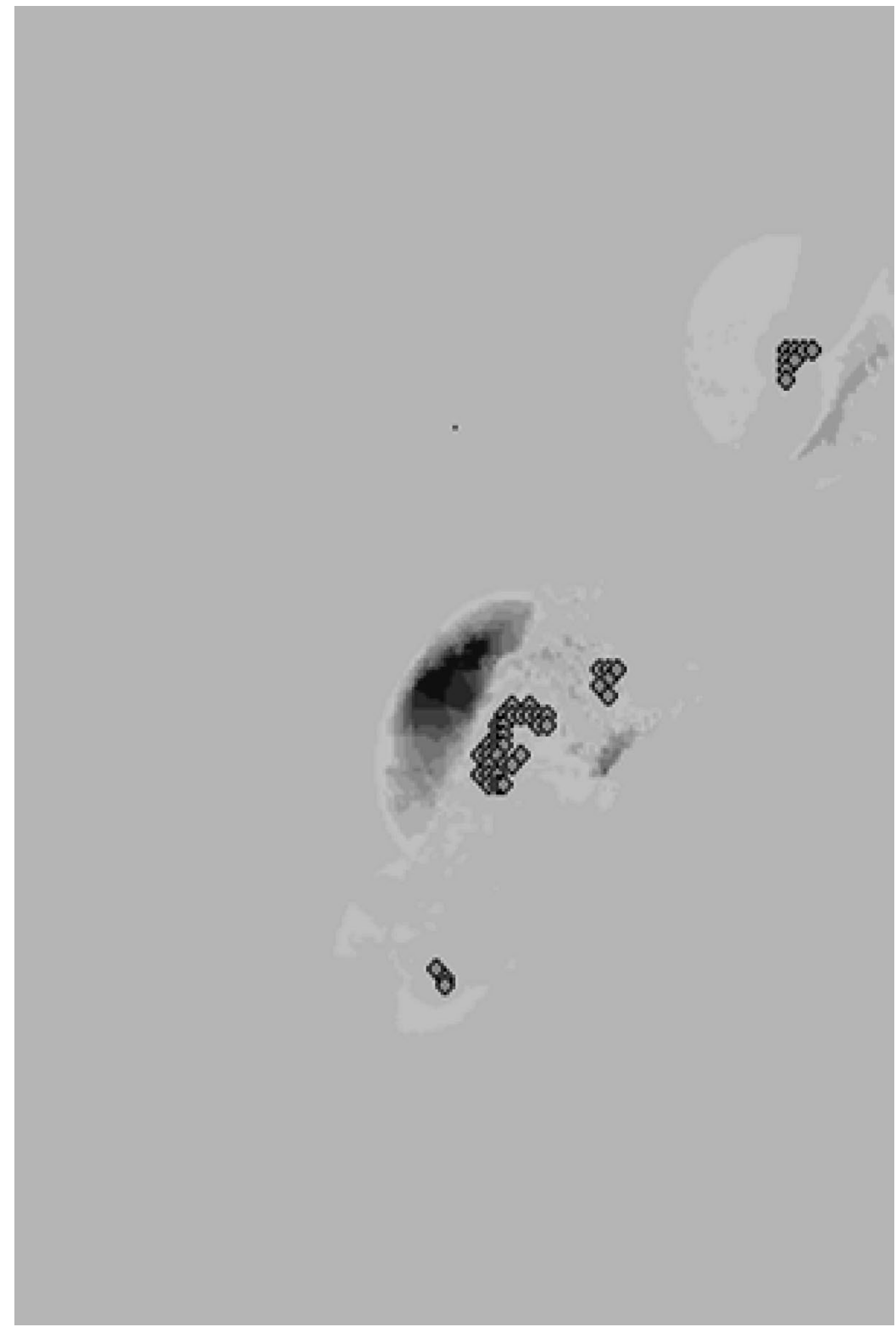

los puntos situados en el borde del territorio, pues en su análisis consideran las zonas dentro del territorio de estudio, pero no las de su entorno exterior. El proceso de análisis se realiza para dos intervalos de distancias: la distancia media (1-3 km) y la de fondo (3-10 $\mathrm{km})$. Se ha despreciado la distancia corta (0-1 $\mathrm{km})$, por no ser significativa, al ser siempre visible el entorno inmediato (43).

El modelo de visibilidad planteado, así como la programación realizada de los macros, tiene una utilidad real práctica de importancia, puesta de manifiesto a la hora del diseño de los modelos de capacidad e impacto.
2. Resultados de visibilidad para $\mathrm{p}_{14}$ En azul se muestran los puntos de $p_{14}$ Las zonas de visibilidad de tonalidad más oscura son las que divisan mayor cantidad de puntos $\mathrm{p}_{14}$. 


\section{BIBLIOGRAFÍA}

(1) Aguiló, M. 2005. "De factor de progreso, al impacto ambiental y al conflicto mediático". Informes de la Construcción, vol. 57 n499-500 pp.87-96.

(2) Otero, l. et al. 2006. "Modelo de localización de áreas potencialmente urbanizables: aplicación al Corredor del Henares." Ciudad y Territorio, vol. 148 pp.331-352.

(3) Otero, I. et al. 2006. "La carretera como elemento de valor paisajístico y ambiental. Captación del valor del paisaje a través de la carretera." Informes de la Construcción, vol. 58 n 504 pp. 39.54.

(4) Lovejoy, D. (Ed.), 1973. "Land use and landscape planning." Leonard Hill, London.

(5) Zube, E.H., y col., 1974. "Perception and measurement of scenic resources in the Southern Connecticut River Valley." Inst. for Man and His Environ, Pub. R-74-1. University of Massachusetts, Amherst, Mass.

(6) Weddle, A. E. and Pickard, J., 1969. "Landscape evaluation, and least social cost evaluation." J.R. Town Plann. Inst., 55: 387-389 y Weddle, A.E., 1969. Landscape with figures. The Town Planning Review, 39, 4, pp 307-318.

(7) Elsner, G.H., y Travis, M.R., 1976. "The role of landscape analytics in landscape planning." USDA Forest Service Gen. Tech. Rep. SE US Southeast For Exp. Stn., 9, pp. 74-87.

(8) Otero et al. 2004. "Guía para la elaboración de estudios de medio físico: Contenido y metodología." Ministerio de Medio Ambiente.

(9) Anile, M. A., Furno, P., Gallo, G., Massolo, A., 2003. "A fuzzy approach to visibility maps creation over digital terrains." Fuzzy Sets and Systems, 135, pp. 63-80.

(10) Kent, M., 1986. "Visibility Analysis of Mining and Waste Tipping Sites- A Review." Landscape and Urban Planning, 13,101-110.

(11) Cohen, D., Shaked, A., 1993. "Photo-realistic imaging of digital terrains. Comput." Graphics Forum 12 (3) (1993) 363-373

(12) Litton, R.B., 1973. "Landscape Control Points: A procedure for Predicting and Monitoring visual Impacts." USDA Forest Service Research Paper PSW-91. Berkeley, California.

(13) Hebblethwaite, R.L., 1970. "Landscape Assessment: Qualitative Zones. Typescrip, C.E.G.B., London y Hebblethwaite, R.L., 1973. Landscape assessment and classification techniques." In: D. Lovejoy (Editor), Land Use and Landscape Planning, $1^{\text {st }}$ edn. Leonard Hill, Aylesbury, pp. 19-50.

(14) Weddle, A. E., 1973. "Applied analysis and evaluation techniques." In: D. Lovejoy (Editor), Land Use and Landscape Planning, $1^{\text {st }}$ edn. Leonard Hill, Aylesbury,pp. 53-82

(15) Tandy, C.R.V., 1971. "The ISOVIST Method of Visual Analysis of Landscape". Land Use Consultants, London.

(16) Van der Ham, R.J.I.M. and Iding, J.A.M.E., 1971. "A Landscape Typology System based on Visual Elements." Department of Landscape Architecture, Agricultural University, Wageningen, The Netherlands.

(17) Department of the Environment, 1976. "Assessment of Major Industrial Applications": A Manual. Department of Environmental Research Report, 13.

(18) Clark, D.B. et al., 1976. "Assessment of Major Industrial Applications: A Manual". Department of the Environment Research Report, 13, London.

(19) Clark, B.D., Chapman, K., Bisset, R: and Wathern, P. 1979. "Environmental impact analysis." In: D. Lovejoy (Editor), Land Use and Landscape Planning, 2nd edn. Leonard Hill, Aylesbury, pp. 51-88.

(20) MacDougall, E.B., 1983. "Microcomputers in Landscape Architecture." Elsevier, New York.

(21) Travis, M.R., Elsner, G:H., Iverson, W.D. and Johson, C:G., 1975. "VIEWIT: Computation of Seen Areas, Slope and Aspect for Land-Use Planning." Pacific Southwest Forest and Range Experimental Station, Berkeley, U.S.A.

(22) Anderson, L. Mosier, J. and Chandler, G. 1980. "Visual management support system." In: Elsner and R.C. Smardon (Editors), Our National Landscape. Proceedings of a Conference on Applied Techniques for Analysis and Management of the Visual Resource, General Technical Report PSW35, Pacific Southwest Forest and Range Experimental Station, Berkeley, CA, pp. 189-195.

(23) Steinitz, C. et al., 1974. "The interaction between urbanization and land. Quality and quantity in environmental planning and design." Harvard University, Cambridge.

(24) Aylward, G. And Turnbul, M., 1977. "Visual analysis: a computer-aided approach to determine visibility." Comput. Aided Des., 9: 103-108 ; Aylward, G. And Turnbul, M., 1978. "Visual analysis: the development and use of descriptors." Des. Methods Theories, 12: 72-88;Aylward, G. And Turnbul, M., 1980. "Introduction to Design Innovations Research. Design Innovations Research," Glasgow ; Aylward, G. And Turnbul, M., 1982. Visual impact analysis. In: A. Piper (Editor), CAD82. $5^{\text {th }}$ International Conference and Exhibition on Computers in Design Engineering, Butterworths, London, pp. 228-238.

(25) Tlusty, W., 1980. "The use of WIEWI and perspective plot to assist in determining the landscape visual absorption capacity." In: G.H. Elsner and R.C. Smardon (Editors), Our National Landscape. Proceedings of a Conference on Applied Techniques for Analysis and Management of the Visual Resource, General Technical Report PSW35, Pacific Southwest Forest and Range Experimental Station, Berkeley, CA, pp. 201-208.

(26) McAulay, I., 1984. "Computer-Aided Visual Impact Analysis of Development in Rural Landscapes." M.Phil. Thesis, Department of Architecture, Plymouth Polytechnic, 142 pp. 
(27) Felleman, J.P., 1982. "Visibility mapping in New York's coastal zone: a case study of alternative methods." Coastal Zone Manage. J., 9: 249-270.

(28) Marshall, R., Wilson, R. and Carlson, W., 1980. "Procedures for generating 3-dimensional terrain." Comput. Graphics, 14: 154-162.

(29) De Floriani, L. and Magillo, P., 1994. "Visibility algorithms on triangulated digital terrain models", Internat. J. GIS 8 (1) pp. 13-41.

(30) Sansoni, C., 1996. "Visual analysis: a new probabilistic technique to determine landscape visibility, Comput." Aid. Design 28 (4) pp. 298-299.

(31) Coventry-Solihull-Warwickshire, 1971. "A strategy for the Sub-Region." Supplementary Rep. 5, Countryside County Planning Department, Warwick.

(32) Steinitz, C., 1979. "Simulating alternative policies for implementing the Massachusetts scenic and recreational rivers act: The north river demostration project." Landscape planning, 6, pp. 51-89.

(33) Kent, M., 1979. "Regional Evaluation for Colliery Spoil Reclamation." Ph.D. Thesis, Department of Landscape Architecture, University of Sheffield, unpublished, 291 pp.

(34) Felleman, J.P., 1979. "Landscape Visibility Mapping: Theory and Practice." College of Environmental Science and Forestry, State University of New York.

(35) Ramos, A., Ramos, F., Cifuentes, P. and Fernández-Canadas, M. 1976. "Visual landscape evaluation, a grid technique." Landscape Plann., 3: 67-88.

(36) Blanco, A., Gonzales, S. And Ramos A., 1982. "Visual landscape classification in the coastal strip of Santander." Coastal Zone Manage. J., 9: 271-285.

(37) De Veer, A.A. and Burrough, P.A., 1978. "Physiognomic landscape mapping in The Netherlands." Landscape Plann, 5: 45-62.

(38) Burrough, P.A. and De Veer, A.A., 1984. "Automated production of landscape maps for physical planning in The Netherlands", Landscape Plann., 11: 205-226.

(39) Nickerson, D.B., 1980. "Perspective Plot: an Interactive Technique for the Visual Modelling of Land Management Techniques." Forest Service, United States Deparment of Agriculture, Division of Timber Management, Portland, OR.

(40) Miller, D., 2000. "A method for estimating changes in the visibility of land cover." Landscape and Urban Panning 54, pp. 91-104.

(41) Fines, K.D., 1968. "Landscape Evaluation: A research project in East Sussex." Regional Studies, 2, pp. 41-55.

(42) Jones, G.R. et al., 1975. "A method for the quantification of aesthetic values for environmental decision making. Nuclear."

(43) Aramburu Maqua, M.P.; Escribano Bombín, R.; Ramos Gonzalo, L.; Rubio Maroto, R., 2003. "Cartografía del paisaje de la Comunidad de Madrid." Consejería de Medio Ambiente de la Comunidad de Madrid. Madrid.

(44) Elsner, G.H., 1971. "Computing Visible Areas from Proposed Recreational Developments, A Case Study. Pacific Southwest Forest and Range Experimental Station". California. 